\title{
The initial luminosity and mass functions of Galactic open clusters
}

\author{
Hans Zinnecker ${ }^{1}$, Anatoly E. Piskunov ${ }^{2}$, Nina V. Kharchenko ${ }^{3}$, \\ Siegfried Röser ${ }^{4}$, Elena Schilbach ${ }^{4}$, and Ralf-Dieter Scholz ${ }^{1}$ \\ ${ }^{1}$ Astrophysical Institute Potsdam, \\ An der Sternwarte 16, D-14482 Potsdam, Germany \\ email: hzinnecker@aip.de \\ email: rdscholz@aip.de \\ ${ }^{2}$ Institute of Astronomy of the Russian Academy of Sciences, \\ 48 Pyatnitskaya St. 119017, Moscow, Russia \\ email: piskunov@inasan.rssi.ru \\ ${ }^{3}$ Main Astronomical Observatory, National Academy of Sciences of Ukraine, \\ 27 Akademika Zabolotnoho St., 03680 Kyiv, Ukraine \\ email: nkhar@mao.kiev.ua \\ ${ }^{4}$ Ruprecht-Karls-Universität Heidelberg, Astronomisches Rechen-Institut, \\ Mönchhofstr. 12-14, D-69120 Heidelberg, Germany \\ email: roeser@ari.uni-heidelberg.de \\ email: elena@ari.uni-heidelberg.de
}

\begin{abstract}
We have derived a complete magnitude-limited sample of 440 Galactic open clusters in the solar neighborhood, with integrated V-magnitude brighter than 8 mag. This sample can be used to infer the present-day luminosity and mass functions of open clusters up to a given age; it can even be used to construct the initial mass and luminosity function (IMF, ILF) of clusters (defined as visible clusters with age $4-8 \mathrm{Myr}$ ). The high-mass end of the cluster IMF is a power-law with a slope of -2 or slightly shallower $(-1.7)$ while the luminous cluster ILF has a power-slope of -1 , in agreement with what is found for extragalactic clusters. Both distribution functions show a turnover, starting at $300 \mathrm{M}_{\odot}$ and integrated magnitude $-3 \mathrm{mag}$, respectively. The overall birthrate of clusters is 0.4 clusters per $\mathrm{kpc}^{2}$ and per Myr. The average present-day cluster mass is $700 \mathrm{M}_{\odot}$, while the average initial cluster mass is $4500 \mathrm{M}_{\odot}$. The difference of these two average masses indicates the high infant mortality and/or weight loss of Galactic open clusters (due to dynamical evolution).
\end{abstract}

Keywords. open clusters, astrometry, photometry, luminosity function, mass function

\section{Introduction}

This contribution is about a new determination of the present-day and initial luminosity and mass function of open clusters in the nearby Galactic disk, based on a homogeneous cluster data base (Kharchenko et al. 2005a, b) inferred from the ASCC-2.5 bright star $(\mathrm{V}<11.5 \mathrm{mag})$ catalog (Kharchenko 2001). Using astrometric and photometric information of individual stars, this uniform all-sky catalog ultimately provides cluster central positions, kinematics, ages, colors, integrated cluster magnitudes, as well as tidal cluster masses. The masses are derived from fitting 3-parameter King profiles to the radial stellar surface density distribution and thus are independent of the cluster luminosities!

We remind the reader that open clusters are typical representatives of the Galactic disk population and excellent examples to calibrate astronomical distances, reddenings, and ages. Open clusters are also great laboratories for studies of stellar evolution and of 
the frequency of the various stellar types, such as Cepheids, red giants, brown dwarfs, and maybe exo-planets.

The present analysis supersedes the results of an older similar study on Galactic open clusters (Piskunov et al. 2006). We stress that we are not dealing here with the IMF in clusters (e.g. Scalo 1998) but with the IMF of clusters (Piskunov et al. 2008b).

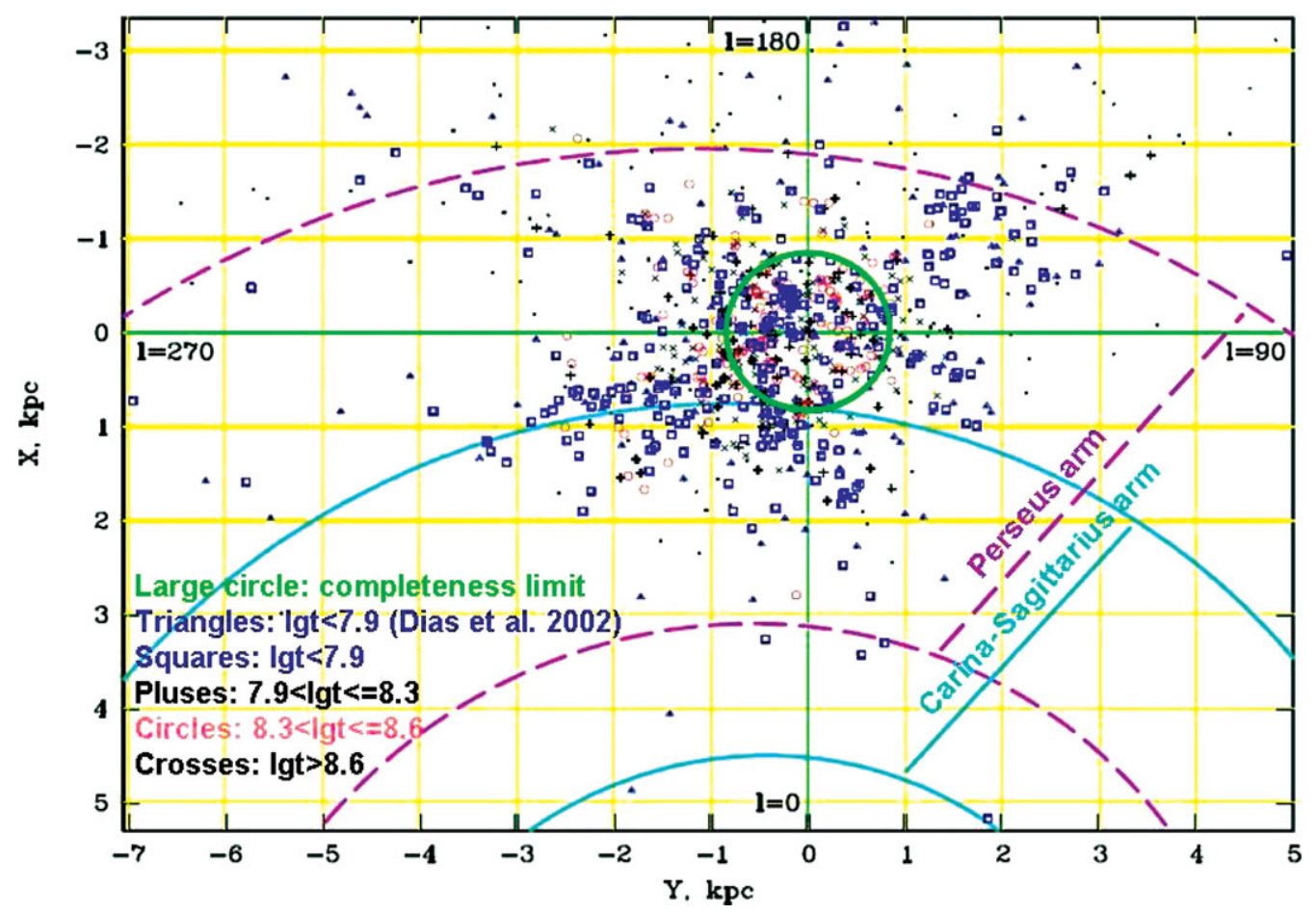

Figure 1. Distribution of open clusters in the $X Y$-plane centered on the location of the Sun. Squares, pluses, circles, and crosses show clusters of different age groups, as indicated in the legend. Dots and triangles mark positions of clusters with known distances and ages from the Dias et al. (2002) catalogue (a somewhat heterogeneous compilation), which are not included in our sample. Long dashed and solid thick curves are the grand design spiral arms with a pitch-angle of $-6^{\circ}$ fitted to young clusters in the Perseus and Carina-Sagittarius regions. The large circle around the Sun indicates the completeness limit of our sample.

\section{The open cluster sample}

The ASCC-2.5 catalog is a homogeneous catalog of 2.5 million stars from which a total of 650 clusters could be extracted (130 of them new, Kharchenko 2005b). Of those, 256 clusters define a volume-limited sample up to $1 \mathrm{kpc}$ and a magnitude limited sample of 440 cluster, with the brightest clusters located beyond $1 \mathrm{kpc}$. Previous open cluster catalogs were published by Becker and Fenkart (1971, 216 clusters observed in UBV), by Lynga $\left(1987,5^{\text {th }}\right.$ Lund catalog), and by Batinelli and Capuzzo-Dolcetta (1991, a subset of 100 bright clusters inside $2 \mathrm{kpc}$ ). All these catalogs are heavily biased, while the ASCC-2.5 optical catalog is free from selection biases and can be used for statistical investigations.

We mention in passing that a list of 100 embedded young clusters was compiled and published in Lada and Lada (2003), while Bica et al. (2003ab) used 2MASS data to discover many new embedded clusters, both in the northern and southern hemisphere 
(see also Froebrich for a systematic 2MASS cluster survey). Also relevant in the context of young clusters is the catalog of 370 Galactic O-stars (GOS v1, v2 - see Maíz-Apellániz et al. 2004, and Sota et al. 2007, respectively; for an early version see Gies 1987). However, we will not make use of it here, but refer to Schilbach and Röser (2008) and their new study of the origin of O-type runaway stars in young clusters.

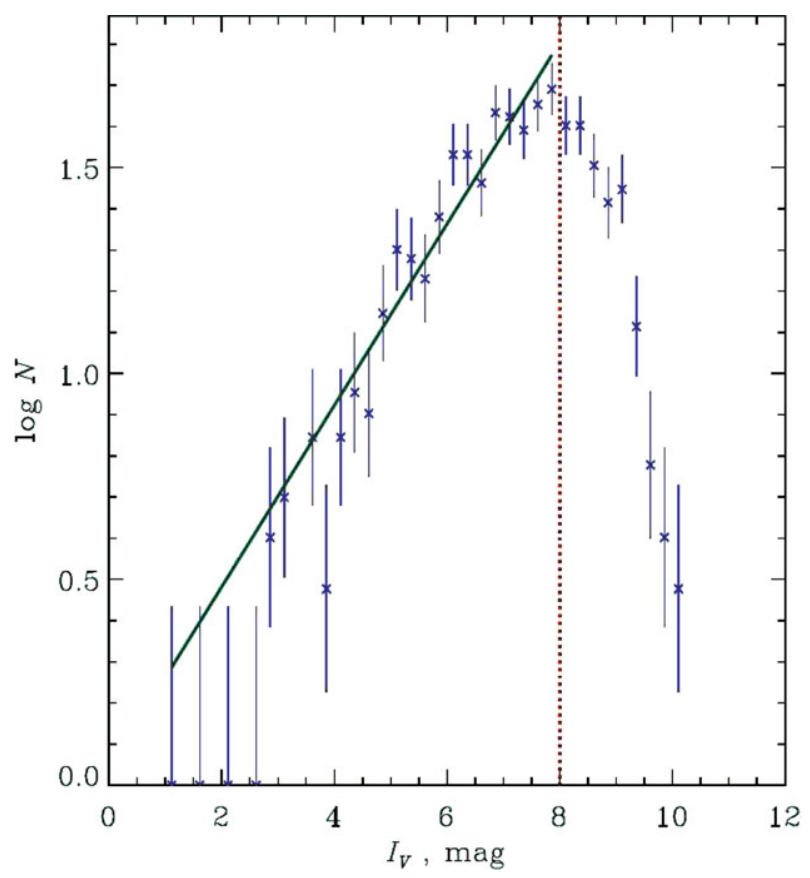

Figure 2. Number distribution of open clusters versus apparent integrated magnitude $\mathrm{I}_{V}$ (crosses). The bars are Poisson errors, the solid line is a linear fit to the distribution and the vertical dotted line is the adopted completeness limit $\mathrm{I}_{V}=8 \mathrm{mag}$.

\section{Determination of open cluster luminosities and masses}

As a measure of cluster brightness needed for the construction of the luminosity function, we take the integrated $V$-magnitudes of each cluster. The integrated magnitudes are computed by summing up the individual fluxes of cluster members selected from the ASCC-2.5 catalogue. Doing so, we avoid contamination by field stars and can thus directly compare our results with observational results of open clusters in other galaxies. To account for the fact that the clusters reside at different distances and show Main Sequences of different lengths in a magnitude-limited survey, we introduce a small (typically $\sim 0.1 \mathrm{mag}$ ) correction for unseen stars $(7$ magnitudes fainter than the bright limit of a cluster). Although small, this correction reduces all the clusters to a common and uniform system of integrated luminosities.

Our cluster masses are derived from "tidal masses" using King's (1962) formula: $\mathrm{M}_{\text {cluster }}=4 \mathrm{~A}(\mathrm{~A}-\mathrm{B}) \mathrm{r}_{t}^{3} / \mathrm{G}$, where $A$ and $B$ are Oort's constants of Galactic differential rotation, $r_{t}$ is the cluster tidal radius, and $G$ is the gravitational constant. Masses depend strongly on the tidal radius which is estimated from a 3-parameter King profile fit to the observed cluster structure together with the cluster core radius and central surface density. Tidal radii peak at about $10 \mathrm{pc}$ and are uncertain by less than $20 \%$. Therefore, cluster masses should be good to about a factor of 2 . For a detailed discussion of tidal 
radii and masses see Piskunov et al. (2007, 2008a). It has been argued that tidal masses may not be applicable to young open clusters but only to globular clusters. This concern is unfounded. As long as star clusters fill their tidal (or Roche) volume, which should be the case after their emergence from the initial embedded cluster stage, everything is fine. The alternative of deriving virial instead of tidal cluster masses (using the cluster velocity dispersion) has so far only be successful in the case of NGC 188 (Geller et al. 2008). Other alternatives to estimate cluster masses, including a scaling with the stellar IMF or using a cluster mass-luminosity relation, have their own problems which we cannot discuss here.
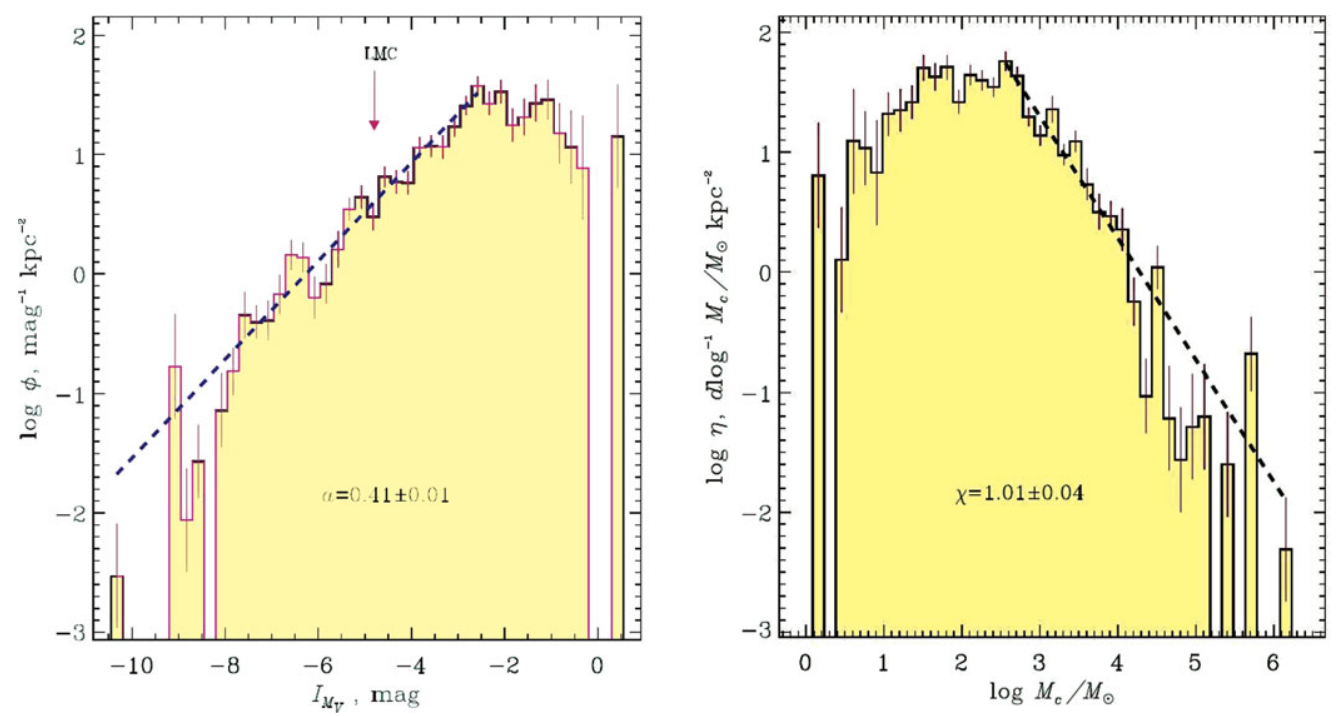

Figure 3. Luminosity (left) and mass (right) functions of Galactic open clusters based on 440 local clusters brighter than the completeness limit $\mathrm{I}_{V}$ of the sample. The bars are Poisson errors, the dashed lines show a linear fit to the brighter/massive parts of the histograms, where $a$ and $\chi$ are the corresponding slopes. The arrow indicates the limit of integrated absolute magnitudes reached for open clusters in the LMC (Larsen 2002).

\section{Cluster luminosity and mass functions}

Fig. 3 shows the present-day luminosity and mass functions that we obtained (CPDLF, CPDMF): they are power-laws at the bright end and turn over towards the faint end (see abstract). Fig. 4 shows the time-resolved, integrated mass functions (dN/dlogM) including the cluster initial mass function (CIMF). The slope of the CIMF at the highmass end (-0.7 at face value) is somewhat uncertain (fit based on just $3-4$ data points) and could be consistent with the slope often quoted for extragalactic clusters ( -1 per unit log mass, or -2 when expressed per unit mass). While some open cluster luminosity functions have been presented before (van den Bergh and Lafontaine 1984, Bhatt et al. 1991, Lata et al. 2002), this is the first CIMF presented for the Milky Way Galactic Disk.

\section{The fraction of field stars from open clusters}

We find that the fraction of nearby disk field stars that originated in Galactic open clusters is around $50 \%$. This result is different from previous studies in which this fraction was derived and cited to be about $10 \%$ (Wielen 1971, Miller and Scalo 1978, Elmegreen 
and Clemens 1985, Janes et al. 1988, Adams and Myers 2001, Lada and Lada 2003). Even the ealier paper by Piskunov et al. (2006) confirmed this low value. At that time, the use of the present-day average open cluster mass $\left(700 \mathrm{M}_{\odot}\right)$ instead of the initial average mass $\left(4500 \mathrm{M}_{\odot}\right)$ led to a factor of 6.5 underestimate which has only been recently corrected in Piskunov et al. (2008b). Also, the birthrate of young open clusters has been revised upward, from $\sim 0.2$ per $\mathrm{kpc}^{2}$ and Myr in Piskunov et al. 2006 to 0.4 per $\mathrm{kpc}^{2}$ and Myr in Piskunov et al. (2008b). One has to multiply the birthrate with the age of the Milky Way Disk $(8-12 \mathrm{Gyr})$ and the average initial cluster mass $\left(4500 \mathrm{M}_{\odot}\right)$ to obtain the surface density of field stars that originated from open clusters: this yields $18 \mathrm{M}_{\odot}$ per pc ${ }^{2}$ as the amount of stellar mass resulting from dissolved open clusters over the age of the Galaxy (assuming a constant cluster formation rate over $10 \mathrm{Gyr}$ ). The local surface mass density in disk stars is $36 \mathrm{M}_{\odot}$ per $\mathrm{pc}^{2}$ (Flynn et al. 2006), thus the ratio is $18 / 36$ or $50 \%$.

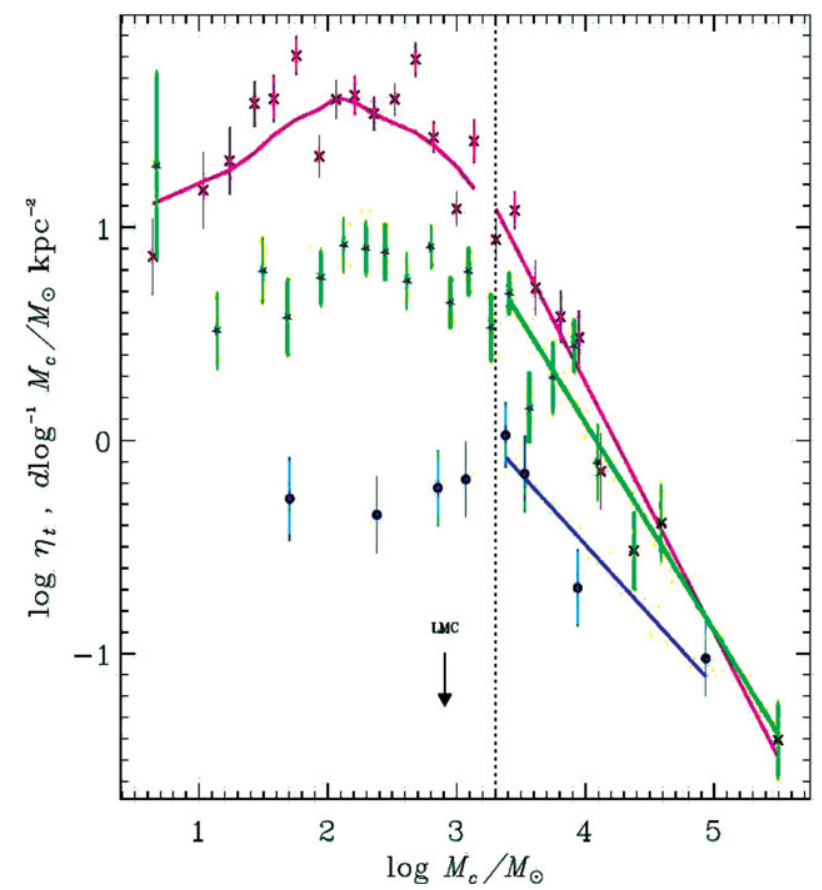

Figure 4. Evolution of the "integrated" mass function of Galactic open clusters (fixed lower age limit and variable upper age limit). Different symbols mark samples with different upper limits of cluster ages. The filled circles are for clusters with $\log t \leqslant 6.9$, the star symbols show cluster mass function for $\log t \leqslant 7.9$, and crosses indicate the CPDMF based on all 440 clusters $(\log t \leqslant 9.5)$. The bars are Poisson errors. The straight lines are the corresponding fits to linear parts of the mass functions at masses greater than $\log M_{c}=3.3$ indicated by the vertical dotted line. The fitted curve at $\log M_{c}<3.3$ is the smoothed CPDMF. The arrow indicates the lower mass limit reached for open clusters in the LMC.

\section{Acknowledgments}

We thank the DFG for a German-Russian collaborative grant (436 RUS 113/757/0-2) to support this study. AEP gratefully acknowledges a grant from the Russian Foundation of Basic Research (RFBR 07-02-91566). HZ is grateful to the SOC for awarding IAU travel money without which this paper could not have been presented. He is also very grateful to U. Hanschur (AIP) for his help to prepare submitting this contribution. 


\section{References}

Adams, F. C. \& Myers, P. C. 2001, ApJ, 553, 744

Battinelli, P. \& Capuzzo-Dolcetta, R. 1991, MNRAS, 249, 76

Becker, W. \& Fenkart, R. 1971, A\&SAS, 4, 241

Bhatt, B. C., Pandey, A. K., \& Mahra, H. S. 1991, JApA, 12, 179

Bica, E., Dutra, C. M., \& Barbuy, B. 2003a, A\&A, 397, 177

Bica, E., Dutra, C. M., Soares, J., \& Barbuy, B. 2003b, A $8 A$, 404, 223

Dias, W. S., Alessi, B. S., Moitinho, A., \& Lépine, J. R. D. 2002, A\&A, 389, 871

Elmegreen, B. G. \& Clemens, C. 1985, ApJ, 294, 523

Flynn, C., Holmberg, J., Portinari, L., Fuchs, B., \& Jahreiss, H. 2006, MNRAS, 372, 1149

Froebrich, D., Scholz, A., \& Raftery, C. L. 2007, MNRAS, 374, 399

Geller, A. M., Mathieu, R. D., Harris, H. C., \& McClure, R. D. 2008, AJ, 135, 2264

Gies, D. R. 1987, ApJS, 64, 545

Janes, K. A., Tilley, C., \& Lynga, G. 1988, AJ, 95, 771

Kharchenko, N. V. 2001, Kinematics and Physics of Celestial Bodies, 17, 409

Kharchenko, N. V., Piskunov, A. E., Röser, S., Schilbach, E. \& Scholz, R.-D. 2005a, A $\& A, 438$, 1163

Kharchenko, N. V., Piskunov, A. E., Röser, S., Schilbach, E., \& Scholz, R.-D. 2005b, A\&A, 440, 403

King, I. 1962, $A J, 67,471$

Lada, C. J. \& Lada, E.,A. 2003, ARAA, 41, 57

Larsen, S. S. 2002, AJ, 124, 1393

Lata, S., Pandey, A. K., Sagar, R., \& Mohan, V. 2002, A\&A, 388, 158

Lyngå, G. 1987, ESO Conf. Workshop Proc., 28, 379

Maíz-Apellániz, J., Walborn, N. R., Galué, H. Á., \& Wei, L. H. 2004, ApJS, 151, 103

Miller, G. E. \& Scalo, J. M. 1978, PASP, 90, 506

Piskunov, A. E. Kharchenko, N. V., Röser, S., Schilbach, E. \& Scholz, R.-D. 2006, A $ॄ$ A, 445, 545

Piskunov, A. E., Schilbach, E., Kharchenko, N. V., Röser, S., \& Scholz, R.-D. 2007, A\&A, 468, 151

Piskunov, A. E., Schilbach, E., Kharchenko, N. V., Röser, S., \& Scholz, R.-D. 2008a, A 6 A 477, 165

Piskunov, A. E., Kharchenko, N. V., Schilbach, E., Röser, S., Scholz, R.-D., \& Zinnecker, H. $2008 \mathrm{~b}, A E \mathcal{E} A$, accepted

Schilbach, E. \& Röser, S. 2008, A\& $A$, accepted

Scalo J. 1998, The Stellar Initial Mass Function (38th Herstmonceux Conference) eds. G. Gilmore and D. Howell. ASP Conference Series, Vol. 142, 1998, p.201

Sota, A., Maíz-Apellániz, J., Walborn, N. R., \& Shida, R. Y. 2007, astro-ph/0703005

van den Bergh, S. \& Lafontaine, A. 1984, AJ, 89, 1822

Wielen, R. 1971, A\&A, 13, 309

\section{Discussion}

K. Freeman: Where do the other $50 \%$ of the field stars form?

H. Zinnecker: In OB associations (like Sco-Cen) and T associations (like Taurus). 